\title{
Iterative and Iterative-Noniterative Integral Solutions in 3-Loop Massive QCD Calculations*
}

\author{
J. Ablinger, C.-S. Radu, and C. Schneider \\ Research Institute for Symbolic Computation (RISC), Johannes Kepler University, \\ Altenbergerstraße 69, A-4040, Linz, Austria
}

\section{A. Behring,}

Institut für Theoretische Teilchenphysik und Kosmologie, RWTH Aachen University, Sommerfeldstr. 16, D-52074 Aachen, Germany

\section{J. Blümlein; A. De Freitas}

Deutsches Elektronen-Synchrotron, DESY, Platanenallee 6, D-15738 Zeuthen, Germany

\section{E. Imamoglu, M. van Hoeij}

Department of Mathematics, Florida State University, 208 Love Building, 1017 Academic Way, Tallahassee, FL 32306-4510, USA

\section{A. von Manteuffel}

Department of Physics and Astronomy, Michigan State University, East Lansing, MI 48824, USA

\section{C.G. Raab}

Institute for Algebra, Johannes Kepler University, Altenbergerstraße 69, A-4040 Linz, Austria

Various of the single scale quantities in massless and massive QCD up to 3-loop order can be expressed by iterative integrals over certain classes of alphabets, from the harmonic polylogarithms to root-valued alphabets. Examples are the anomalous dimensions to 3-loop order, the massless Wilson coefficients and also different massive operator matrix elements. Starting at 3-loop order, however, also other letters appear in the case of massive operator matrix elements, the so called iterative non-iterative integrals, which are related to solutions based on complete elliptic integrals or any other special function with an integral representation that is definite but not a Volterratype integral. After outlining the formalism leading to iterative non-iterative integrals,we present examples for both of these cases with the 3-loop anomalous dimension $\gamma_{q g}^{(2)}$ and the structure of the principle solution in the iterative non-interative case of the 3-loop QCD corrections to the $\rho$-parameter.

RADCOR 2017 - 13th International Symposium on Radiative Corrections (Applications of Quantum Field Theory to Phenomenology) 24-29 September 2017, St. Gilgen, Austria

* This work was supported in part by the Austrian Science Fund (FWF) grant SFB F50 (F5006-N15, F5009-N15), FWF-Grant P 27229, the European Commission through contract PITN-GA-2012-316704 (HIGGSTOOLS), and National Science Foundation under Grant No. 1719863.

${ }^{\dagger}$ Speaker 


\section{The Functional Structure of Feynman Integrals in the Single Scale Case}

We will consider single scale quantities in massless and massive QCD up to 3-loop order. They are given by Feynman parameter integrals that depend only on one dimensionless parameter. Examples are the anomalous dimensions and the massless and massive Wilson coefficients, as well as the associated massive operator matrix elements (OMEs), which can be represented by special functions of certain kinds. These are the iterative integrals up to the 2-loop case, which are given by

$$
H_{f_{b}, \vec{f}_{a}}(x)=\int_{0}^{x} d y f_{b}(y) H_{\vec{f}_{a}}(y), f_{c_{i}}(z) \in \mathfrak{A}, H_{\emptyset}=1,
$$

with $\mathfrak{A}$ some alphabet of functions or distributions $f_{c_{i}}(z)$, or the related nested sums in Mellin $N$ space obtained by performing a Mellin transformation

$$
\mathbf{M}\left[H_{\vec{f}_{a}}(y)\right](N)=\int_{0}^{1} d y y^{N-1} H_{\vec{f}_{a}}(y) .
$$

The alphabet $\mathfrak{A}$ covers the harmonic polylogarithms [1], Kummer-Poincaré iterated integrals [2], cyclotomic harmonic polylogarithms [3], root-valued iterated integrals [4], or generalizations thereof. In Mellin space the nested sums can be expressed in terms of the harmonic sums [5] and nested sums of Refs. [2-4] correspondingly.

All of these solutions have in common that their associated differential or difference equations are factorizable to first order. They, or the difference equations obtained by using a formal power series Ansatz in solving the differential equations, can be completely solved using difference field and ring theory [6-14], as has been described in detail in Ref. [15]. In the case of master integrals, this is possible for whatsoever basis. The corresponding alphabet $\mathfrak{A}$ is constructively found in this way. These algorithms are implemented in the packages Sigma [16,17], EvaluateMultisums and SumProduction [18]. In various places the package HarmonicSums [2-4,19,20] is used to operate on special functions of various kind emerging throughout the calculation.

Beginning at 3-loop order a series of Feynman or master integrals obey differential equations which do not factorize at first order order anymore for the $O\left(\varepsilon^{k}\right)$ terms for $k \geq 0, k \in \mathbb{N}$, while for $k<0$, first order factorization holds. This again can be proven by using the package Sigma, which finds all the first order factors and will reduce the corresponding problem by leading to a remainder difference (differential) equation of order $o>1$.

The first new structures appear in the form of non-factorizing second order differential equations

$$
\left[\frac{d^{2}}{d x^{2}}+p(x) \frac{d}{d x}+q(x)\right] f(x)=N(x)
$$

containing usually more than three singularities and rational functions $p(x), q(x) \in \mathbb{Q}(x)$. Eq. (1.3) can be a Heun [21] or a more general differential equation, i.e. more general than a Gauß differential equation. Yet one may find ${ }_{2} F_{1}(a, b ; c ; z)$ homogeneous solutions, with rational parameters $a, b, c$ and $z(x) \in \mathbb{Q}(x)$. In even more general cases, one will have non-factorizable differential equations with rational coefficients of even higher order. Their solution is given in general by a 
combination of (known or yet unknown) higher transcendental functions. As we have outlined in studying general single scale Feynman diagrams in [22], the solutions can be obtained in the form of (multiple) Mellin-Barnes [23] integrals. These integrals $F[r(y)]$ depend on one variable $y$, in such a way that the integral cannot be rewritten such that $y$ appears in the integration bounds only. Subsequently, further integrals shall be performed in the variable $y$ multiplying with other integrals of this type and iterative integrals. In this way, non-iterative letters appear emerging in so-called iterative non-iterative integrals, [24],

$$
\begin{aligned}
\mathbb{H}_{a_{1}, \ldots, a_{m-1} ;\left\{a_{m} ; F_{m}\left(r\left(y_{m}\right)\right)\right\}, a_{m+1}, \ldots, a_{q}}(x)= & \int_{0}^{x} d y_{1} f_{a_{1}}\left(y_{1}\right) \int_{0}^{y_{1}} d y_{2} \ldots \int_{0}^{y_{m-1}} d y_{m} f_{a_{m}}\left(y_{m}\right) F_{m}\left[r\left(y_{m}\right)\right] \\
& \times H_{a_{m+1}, \ldots, a_{q}}\left(y_{m+1}\right),
\end{aligned}
$$

and $F[r(y)]$ is given by

$$
F[r(y)]=\int_{0}^{1} d z g(z, r(y)), \quad r(y) \in \mathbb{Q}(y) .
$$

Writing the solutions in the case of second order differential equations as ${ }_{2} F_{1}$ solutions has the advantage that a lot more is known about their functional properties, and all the Kummer relations and the contiguous relations can be applied. If and only if it is possible, after using these relations, in order to map the set $\{a, b ; c\}$ into a certain finite set of triples, cf. [24], there is a finite set of functional transformations of the ${ }_{2} F_{1}$ solutions into complete elliptic integrals.

In the ${ }_{2} F_{1}$ solutions having appeared so far in single scale Feynman diagram calculations to higher order [25-42], one could always find elliptic integral representations with either the appearance of only the complete elliptic integral of the first kind $\mathbf{K}$ or of both the complete elliptic integrals of the first and second kind $\mathbf{K}$ and $\mathbf{E}$ in the single scale case. The solutions of the second order differential equations read

$$
\psi(x)=\psi_{1}^{(0)}(x)\left[C_{1}-\int d x \psi_{2}^{(0)}(x) n(x)\right]+\psi_{2}^{(0)}(x)\left[C_{2}+\int d x \psi_{1}^{(0)}(x) n(x)\right]
$$

were $\psi_{1,2}^{(0)}(x)$ are the homogeneous solutions, $n(x)$ denotes the ratio of the inhomogeneity $N(x)$ and the Wronskian $W(x) . C_{1}$ and $C_{2}$ are constants depending on the physical problem.

One can now attempt to diagonalize (1.6) by using modular functions, cf. e.g. [43], or in more special cases, modular forms. To do this one introduces the variable $q$

$$
q=\exp [-\pi \mathbf{K}(1-z(x)) / \mathbf{K}(z(x))]
$$

The original kinematic variable $x$ and all other building blocks of the inhomogeneous solution are then expressed in $q .{ }^{1}$ To find the representation of $x=x(q)$ usually requires a higher order Legendre-Jacobi transformation, cf. $[32,44]$ through which $x(q)$ can be written as a rational term of powers of Dedekind $\eta$ functions $\eta(k \tau), k \in \mathbb{N} \backslash\{0\}, \tau=\ln (q) / i$, [45], with

$$
\prod_{k=1}^{m} \eta^{l_{k}}(k \tau), \quad l_{k} \in \mathbb{Z} .
$$

\footnotetext{
${ }^{1}$ In the cases studied so far the inhomogeneities were always expressible in terms of harmonic polylogarithms. As the $q$ representation depends on the process, the representation of the harmonic polylogarithms have to be calculated for each case newly.
} 
Each of the ratios (1.8), being modular functions under some congruence subgroup of $\Gamma(N)$ for some $N \in \mathbb{N}$, can now be written taking a factor $1 / \eta^{n}(\tau)$ out for some $n \in \mathbb{N}$, such that the remainder term is a modular form. For all of these modular forms one may construct now a finite dimensional basis representation [43] through Lambert-Eisenstein series [46, 47] and products thereof [48]. They are of the form

$$
\begin{aligned}
T_{m, n, l, a, b} & :=\sum_{k=0}^{\infty} \frac{(m k+n)^{l-1} q^{a(m k+n)}}{1-q^{b(m k+n)}} \\
& =n^{l-1} q^{n(a-b)} \operatorname{Li}_{0}\left(q^{n b}\right)+q^{n(a-b)} \sum_{j=0}^{l-1}\left(\begin{array}{c}
l-1 \\
j
\end{array}\right) m^{j} n^{l-1-j} \operatorname{ELi}_{-j ; 0}\left(q^{m(a-b)} ; q^{n b} ; q^{m b}\right)
\end{aligned}
$$

or special cases thereof. Here $\operatorname{Li}_{0}(x)=x /(1-x)$ and $\mathrm{ELi}_{n, m}(x, y ; q)$ denotes the elliptic polylogarithm [37]

$$
\operatorname{ELi}_{n, m}(x, y ; q)=\sum_{k=1}^{\infty} \sum_{l=1}^{\infty} \frac{x^{k}}{k^{n}} \frac{y^{l}}{l^{m}} q^{n m} .
$$

Note that in Eq. (1.9) the parameters $x$ and $y$ of the elliptic polylogarithm (1.10), which are not supposed to depend on $q$, do depend on $q$. One may synchronize arguments $q^{m} \rightarrow q,-q \rightarrow q$ within the notion of elliptic polylogarithms, cf. [24]. In the above sense, the elliptic polylogarithm provides a frame for the result, but not in the original sense. Due to the multiplication relation of the elliptic polylogarithm all of these terms can be represented in the form of elliptic polylogarithms, which are formal power series in $q$.

However, there are still the factors $1 / \eta^{k}(\tau)$ for which the closed form representation of its formal power series in $q$ is yet unknown, unlike its infinite product representation [48]. This means that for the case that all $k=0$, the integral-relation of the elliptic polylogarithms will yield elliptic polylogarithms again. Up to terms of $\ln (q)$ the inhomogeneous solution (1.6) can thus be diagonalized and be written mainly in terms of elliptic polylogarithms. This is different in cases in which $k \neq 0$. Here the $q$-integrals will usually lead to higher transcendental functions in $q$, but will not be diagonalized in operating on $q$ series for which the expansion coefficients are known in closed form.

In the following we will illustrate the above formalisms applied to two important cases. One of them is the 3-loop anomalous dimension, which can be expressed in $x$-space either in terms of iterative integrals, which are harmonic polylogarithms, or the nested harmonic sums in Mellin $N$-space. The 3-loop QCD corrections to the $\rho$-parameter, on the other hand, are an example in which elliptic integrals are present. At least in intermediary steps one needs modular functions, represented by modular forms as well as a pre-factor $1 / \eta^{k}(\tau)$.

\section{The 2-loop anomalous dimension $\gamma_{q g}^{(2)}$}

In Ref. [49] we calculated the 3-loop anomalous dimension $\gamma_{q g}^{(2)}(N)$ from first principles in a massive environment. It has been obtained from the $O(1 / \varepsilon)$ term of the unrenormalized 3-loop OME $\hat{\hat{A}}_{Q g}^{(3)}$. Due to the polynomial dependence of the master integral on the dimensional parameter $\varepsilon$, one would also encounter elliptic terms if the calculation of the master integrals would be carried out 
directly. The computational method we used was, however, the method of arbitrarily large fixed moments [51]. In this way, new higher transcendental functions of whatsoever complexity will map onto specific series of rational numbers. These series are united to a series for $\hat{A}_{Q g}^{(3)}$. Due to this, we obtain new series in $N$ for the $O(1 / \varepsilon)$ term, for all the individual color- $\zeta$ factors labeled by the corresponding Casimir operators and potential multiple zeta values [50]. We have generated 2000 moments for $\hat{\hat{A}}_{Q g}^{(3)}$ using the formalism of Ref. [51] in Ref. [49]. Guessing methods [52, 53] now allow to find a difference equation for the different contributions to $\gamma_{q g}^{(2)}(N)$. It turns out that this equation, unlike the ones for the master integrals, which would be needed to higher powers in $\varepsilon$, factorizes all in first order. They can be solved by applying difference field and ring methods [6-14] using the packages Sigma [16,17], EvaluateMultiSums and SumProduction [18]. Here the package HarmonicSums $[2-4,19,20]$ is used to operate on special functions of various kind.

We obtain

$$
\begin{aligned}
& \gamma_{q g}^{(2)}=C_{A} N_{F}^{2} T_{F}^{2}\left\{-\frac{128\left(5 N^{2}+8 N+10\right)}{9 N(N+1)(N+2)} S_{-2}-\frac{64 P_{8}}{9 N(N+1)^{2}(N+2)^{2}} S_{1}^{2}\right. \\
& -\frac{64 P_{9}}{9 N(N+1)^{2}(N+2)^{2}} S_{2}+\frac{64 P_{25}}{27 N(N+1)^{3}(N+2)^{3}} S_{1}+\frac{16 P_{34}}{27(N-1) N^{4}(N+1)^{4}(N+2)^{4}} \\
& \left.+p_{q g}^{(0)}(N)\left(\frac{32}{9} S_{1}^{3}-\frac{32}{3} S_{1} S_{2}+\frac{64}{9} S_{3}+\frac{128}{3} S_{-3}+\frac{128}{3} S_{2,1}\right)\right\} \\
& +C_{F} N_{F}^{2} T_{F}^{2}\left\{\frac{32\left(5 N^{2}+3 N+2\right)}{3 N^{2}(N+1)(N+2)} S_{2}+\frac{32\left(10 N^{3}+13 N^{2}+29 N+6\right)}{9 N^{2}(N+1)(N+2)} S_{1}^{2}\right. \\
& -\frac{32 P_{12}}{27 N^{2}(N+1)^{2}(N+2)} S_{1}+\frac{4 P_{38}}{27(N-1) N^{5}(N+1)^{5}(N+2)^{4}} \\
& \left.+p_{q g}^{(0)}(N)\left(-\frac{32}{9} S_{1}^{3}-\frac{32}{3} S_{1} S_{2}+\frac{320}{9} S_{3}\right)\right\} \\
& +C_{A} C_{F} N_{F} T_{F}\left\{-128 \frac{N^{3}-7 N^{2}-6 N+4}{N^{2}(N+1)^{2}(N+2)} S_{-2,1}+\frac{32 P_{5}}{N^{2}(N+1)^{2}(N+2)} S_{-3}\right. \\
& +\frac{16 P_{18}}{9(N-1) N^{2}(N+1)^{2}(N+2)^{2}} S_{1}^{3}-\frac{16 P_{24}}{9(N-1) N^{2}(N+1)^{2}(N+2)^{2}} S_{3} \\
& -\frac{8 P_{27}}{9(N-1) N^{3}(N+1)^{3}(N+2)^{2}} S_{1}^{2}+\frac{8 P_{29}}{3(N-1) N^{3}(N+1)^{3}(N+2)^{3}} S_{2} \\
& +\frac{P_{37}}{27(N-1) N^{5}(N+1)^{5}(N+2)^{4}}+p_{q g}^{(0)}(N)\left[\left(\frac{640}{3} S_{3}-384 S_{2,1}\right) S_{1}+\frac{32}{3} S_{1}^{4}\right. \\
& +160 S_{1}^{2} S_{2}-64 S_{2}^{2}+\left(192 S_{1}^{2}+64 S_{2}\right) S_{-2}+96 S_{-2}^{2}+224 S_{-4}-64 S_{2,-2}+64 S_{3,1} \\
& \left.+192 S_{2,1,1}-256 S_{-2,1,1}-192 S_{1} \zeta_{3}\right]-\frac{192 P_{17}}{(N-1) N^{2}(N+1)^{2}(N+2)^{2}} \zeta_{3} \\
& +\left(\frac{16 P_{16}}{3(N-1) N^{2}(N+1)^{2}(N+2)^{2}} S_{2}+\frac{16 P_{35}}{27(N-1) N^{4}(N+1)^{4}(N+2)^{4}}\right) S_{1}
\end{aligned}
$$




$$
\begin{aligned}
& +\left[-\frac{32 P_{15}}{N^{3}(N+1)^{3}(N+2)}+\frac{128\left(N^{3}-13 N^{2}-14 N-2\right)}{N^{2}(N+1)^{2}(N+2)} S_{1}\right] S_{-2} \\
& \left.+\frac{96 N(N+1) p_{q g}^{(0)}(N)^{2}}{N-1} S_{2,1}\right\} \\
& +C_{A}^{2} N_{F} T_{F}\left\{-\frac{64 P_{11}}{(N-1) N^{2}(N+1)^{2}(N+2)^{2}} S_{-2,1}-\frac{16 P_{20}}{9(N-1) N^{2}(N+1)^{2}(N+2)^{2}} S_{3}\right. \\
& -\frac{32 P_{21}}{3(N-1) N^{2}(N+1)^{2}(N+2)^{2}} S_{-3}-\frac{8 P_{22}}{9(N-1) N^{2}(N+1)^{2}(N+2)^{2}} S_{1}^{3} \\
& +\frac{16 P_{32}}{9(N-1)^{2} N^{3}(N+1)^{3}(N+2)^{3}} S_{1}^{2}+\frac{16 P_{33}}{9(N-1)^{2} N^{3}(N+1)^{3}(N+2)^{3}} S_{2} \\
& -\frac{8 P_{39}}{27(N-1)^{2} N^{5}(N+1)^{5}(N+2)^{5}}+p_{q g}^{(0)}(N)\left[-\frac{32 P_{10}}{3(N-1) N(N+1)(N+2)} S_{2,1}\right. \\
& +\left(-\frac{704}{3} S_{3}+128 S_{2,1}+512 S_{-2,1}\right) S_{1}-512 S_{-3} S_{1}-\frac{16}{3} S_{1}^{4}-160 S_{1}^{2} S_{2}-16 S_{2}^{2}-32 S_{4} \\
& +\left(-192 S_{1}^{2}+320 S_{2}\right) S_{-2}-96 S_{-2}^{2}+96 S_{-4}-448 S_{2,-2}-128 S_{3,1}+512 S_{-3,1} \\
& \left.-768 S_{-2,1,1}+192 S_{1} \zeta_{3}\right]+\frac{96(N-2)(N+3) P_{4}}{(N-1) N^{2}(N+1)^{2}(N+2)^{2}} \zeta_{3} \\
& +\left(\frac{8 P_{19}}{3(N-1) N^{2}(N+1)^{2}(N+2)^{2}} S_{2}-\frac{8 P_{36}}{27(N-1)^{2} N^{4}(N+1)^{4}(N+2)^{4}}\right) S_{1} \\
& \left.+\left(-\frac{64 P_{13}}{(N-1) N^{2}(N+1)^{2}(N+2)^{2}} S_{1}+\frac{32 P_{30}}{9(N-1) N^{3}(N+1)^{3}(N+2)^{3}}\right) S_{-2}\right\} \\
& +C_{F}^{2} N_{F} T_{F}\left\{\frac{P_{31}}{N^{5}(N+1)^{5}(N+2)}-\frac{8 P_{3}}{3 N^{2}(N+1)^{2}(N+2)} S_{1}^{3}-\frac{16 P_{6}}{3 N^{2}(N+1)^{2}(N+2)} S_{3}\right. \\
& +\frac{64 P_{14}}{N^{3}(N+1)^{2}(N+2)} S_{-2}-\frac{8 P_{23}}{N^{3}(N+1)^{3}(N+2)} S_{1}^{2}+\frac{8 P_{26}}{N^{3}(N+1)^{3}(N+2)} S_{2} \\
& +p_{q g}^{(0)}(N)\left[\left(-\frac{704}{3} S_{3}+256 S_{2,1}\right) S_{1}-256 S_{-3} S_{1}-\frac{16}{3} S_{1}^{4}-48 S_{2}^{2}-160 S_{4}-64 S_{-2}^{2}\right. \\
& \left.-192 S_{-4}-\frac{128}{N(N+1)} S_{2,1}-128 S_{2,-2}+64 S_{3,1}+256 S_{-3,1}-192 S_{2,1,1}\right] \\
& +\frac{96(N-1)\left(3 N^{2}+3 N-2\right)}{N^{2}(N+1)^{2}} \zeta_{3}-256 \frac{2-N+N^{2}}{N^{2}(N+1)(N+2)}\left[S_{-2} S_{1}-S_{-2,1}\right] \\
& \left.+\left(-\frac{8 P_{28}}{N^{4}(N+1)^{4}(N+2)}-\frac{8 P_{7}}{N^{2}(N+1)^{2}(N+2)} S_{2}\right) S_{1}-\frac{128(N-1)}{(N+1)^{2}(N+2)} S_{-3}\right\} \text {. }
\end{aligned}
$$

Here, $C_{A}=N_{c}, C_{F}=\left(N_{c}^{2}-1\right) /\left(2 N_{c}\right), T_{F}=1 / 2$ and $N_{c}=3$ in case of QCD, $\zeta_{k}, k \geq 2, k \in \mathbb{N}$ are the values of Riemann's $\zeta$ function, $P_{i}$ denotes computed polynomials in $N, S_{\vec{a}}$ are the nested harmonic 
sums [5]

$$
S_{b, \vec{a}} \equiv S_{b, \vec{a}}(N)=\sum_{k=1}^{N} \frac{(\operatorname{sign}(b))^{k}}{k^{|b|}} S_{\vec{a}}(k), \quad S_{\emptyset}=1, \quad b, a_{i} \in \mathbb{Z} \backslash\{0\}
$$

and we used the shorthand notation

$$
p_{q g}^{(0)}(N)=\frac{N^{2}+N+2}{N(N+1)(N+2)} .
$$

Despite of the emergence of non-first order factorizable terms in the higher order expansion terms in $\varepsilon$ in the master integrals, the method of Ref. [49] allows to project on the terms which really contribute to $O(1 / \varepsilon)$. Due to this, we could calculate $\gamma_{q g}^{(2)}$ even in a massive environment, without first encountering elliptic contributions which would only cancel in the very last step.

\section{The $\rho$-parameter at $O\left(a_{s}^{3}\right)$}

The 3-loop QCD corrections to the $\rho$-parameter have been calculated in [54]. There, some of the master integrals could not be calculated in closed form, and were determined from the beginning by a power series Ansatz in order to finally derive numerical results. In Ref. [24] we recently found an analytic solution of the equations of the type (1.3). Here the inhomogeneity $N(x)$ is always given in terms of harmonic polylogarithms with rational pre-factors. The Wronskian $W(x)$ is given by a (factorizable) polynomial in $x$. For the homogeneous solutions one finds ${ }_{2} F_{1}$ solutions [55]. Let us consider, as an example, Eq. (2.14) of [24]. Its homogeneous solutions are given by

$$
\begin{aligned}
& \psi_{1 a}^{(0)}(x)=\sqrt{2 \sqrt{3} \pi} \frac{x^{2}\left(x^{2}-1\right)^{2}\left(x^{2}-9\right)^{2}}{\left(x^{2}+3\right)^{4}}{ }_{2} F_{1}\left[\begin{array}{cc}
\frac{4}{3} & \frac{5}{3} \\
2
\end{array} z\right] \\
& \psi_{2 a}^{(0)}(x)=\sqrt{2 \sqrt{3} \pi} \frac{x^{2}\left(x^{2}-1\right)^{2}\left(x^{2}-9\right)^{2}}{\left(x^{2}+3\right)^{4}}{ }_{2} F_{1}\left[\begin{array}{c}
\frac{4}{3} \\
2
\end{array} \frac{5}{3} ; 1-z\right],
\end{aligned}
$$

with

$$
z \equiv z(x)=\frac{x^{2}\left(x^{2}-9\right)^{2}}{\left(x^{2}+3\right)^{3}}
$$

This is not yet a solution in terms of complete elliptic integrals. Applying contiguous relations and the triangle group relations $[24,56]$ one, however, obtains

$$
\begin{aligned}
& \psi_{1 b}^{(0)}(x)=\frac{\sqrt{\pi}}{4 \sqrt{6}}\left\{-(x-1)(x-3)(x+3)^{2} \sqrt{\frac{x+1}{9-3 x}} 2 F_{1}\left[\begin{array}{cc}
\frac{1}{2} & \frac{1}{2} \\
1 &
\end{array}\right]\right. \\
& \left.+\left(x^{2}+3\right)(x-3)^{2} \sqrt{\frac{x+1}{9-3 x}} 2 F_{1}\left[\begin{array}{ll}
\frac{1}{2} & -\frac{1}{2} \\
1
\end{array} ; z\right]\right\} \\
& \psi_{2 b}^{(0)}(x)=\frac{2 \sqrt{\pi}}{\sqrt{6}}\left\{x ^ { 2 } \sqrt { ( x + 1 ) ( 9 - 3 x ) } { } _ { 2 } F _ { 1 } \left[\begin{array}{cc}
\frac{1}{2} & \frac{1}{2} \\
1 & ; 1-z]
\end{array}\right.\right. \\
& \left.+\frac{1}{8} \sqrt{(x+1)(9-3 x)}(x-3)\left(x^{2}+3\right)_{2} F_{1}\left[\begin{array}{c}
\frac{1}{2}-\frac{1}{2} \\
1
\end{array} 1-z\right]\right\} \text {, }
\end{aligned}
$$



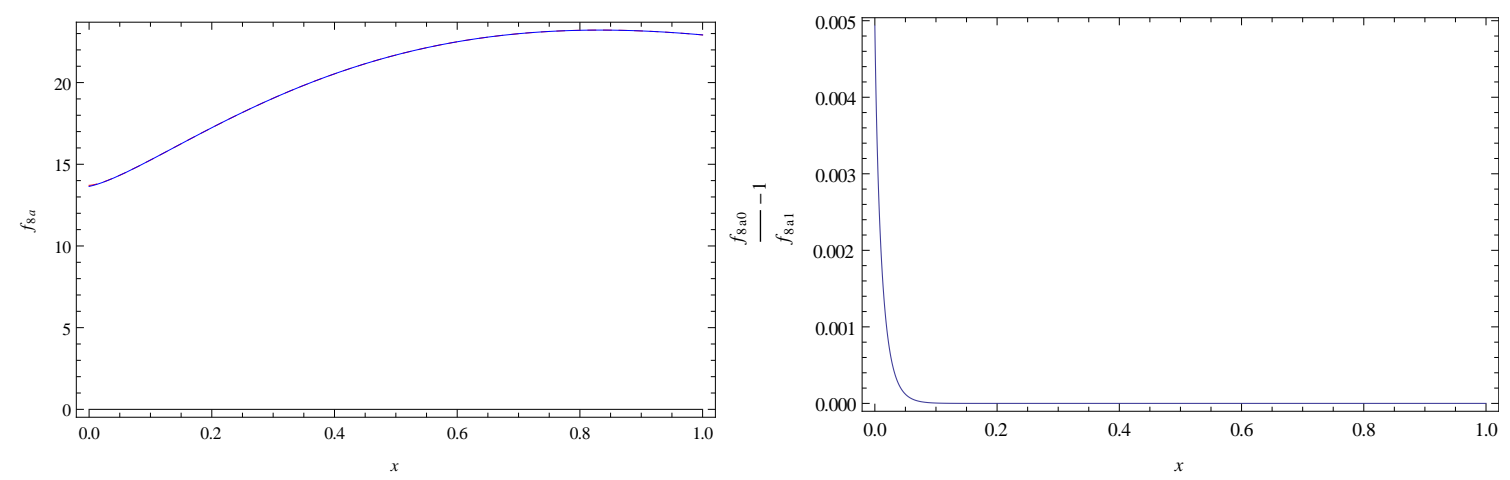

Figure 1: The inhomogeneous solution of Eq. (2.14) of [24] as a function of $x$. Left panel: Red dashed line: expansion around $x=0$; blue line: expansion around $x=1$. Right panel: illustration of the relative accuracy and overlap of the two solutions $f_{8 a}(x)$ around 0 and 1; from Ref. [24].

with

$$
z \equiv z(x)=-\frac{16 x^{3}}{(x+1)(x-3)^{3}}
$$

By using [57]

$$
{ }_{2} F_{1}\left[\begin{array}{cc}
\frac{1}{2} & \frac{1}{2} \\
1 & ; z
\end{array}\right]=\frac{2}{\pi} \mathbf{K}(z), \quad{ }_{2} F_{1}\left[\begin{array}{cc}
\frac{1}{2}-\frac{1}{2} & ; z \\
1
\end{array}\right]=\frac{2}{\pi} \mathbf{E}(z)
$$

one obtains the solution in terms of complete elliptic integrals of the first and second kind and may show that the elliptic integral of the second kind, E, cannot be transformed away. Since the ${ }_{2} F_{1}$ functions have no representation as an integral in which the $z$ dependence appears in the integration bounds only, the inhomogeneous solution (1.6) is an iterative non-iterative integral of the kind of Eq. (1.4), [24].

One may now transform the inhomogeneous solution into power series solutions around $x=0$ and $x=1$ applying standard methods implemented in mathematica and maple to arbitrary order. One obtains an accuracy of the overlapping solutions in the complete range $x \in[0,1]$, e.g. to an accuracy of $O\left(10^{-30}\right)$ by the first fifty expansion terms. We show the solution in Figure 1.

We now would like to derive a $q$ series solution for the same problem. First the kinematic variable $x$ is expressed by $x=-1 / y$,

$$
z(x) \equiv k^{2}=\frac{16 y}{(1-y)(1+3 y)^{3}}=\frac{\vartheta_{2}^{4}(q)}{\vartheta_{3}^{4}(q)}
$$

with $\vartheta_{k}(q), k=1,2,3,4$ the Jacobi $\vartheta$-functions [57]. One solves (3.8) and obtains

$$
x=-\frac{\eta^{4}(2 \tau) \eta^{2}(3 \tau)}{\eta^{2}(\tau) \eta^{4}(6 \tau)}
$$

which is a modular form multiplied by $1 / \eta^{12}(\tau)$ and $\propto 1 / q$.

Some of the building blocks of the inhomogeneous solution are modular forms like

$$
\mathbf{K}(z)=\frac{\pi}{2} \sum_{k=1}^{\infty} \frac{q^{k}}{1+q^{2 k}}=\frac{\pi}{4} \bar{E}_{0 ; 0}(i ; 1 ; q), \quad \text { with }
$$




$$
\bar{E}_{n ; m}(x ; y ; q)=\frac{1}{i}\left[\operatorname{ELi}_{n ; m}(x ; y ; q)-\operatorname{ELi}_{n ; m}\left(x^{-1} ; y^{-1} ; q\right)\right], n+m \text { even }
$$

while others are not. One example is $\mathbf{E}(z(x))$, given by

$$
\mathbf{E}\left(k^{2}\right)=\mathbf{K}\left(k^{2}\right)+\frac{\pi^{2} q}{\mathbf{K}\left(k^{2}\right)} \frac{d}{d q} \ln \left[\vartheta_{4}(q)\right] .
$$

One has

$$
\begin{aligned}
q \frac{\vartheta_{4}^{\prime}(q)}{\vartheta_{4}(q)}= & -\frac{1}{2}\left[\operatorname{ELi}_{-1 ; 0}(1 ; 1 ; q)+\operatorname{ELi}_{-1 ; 0}(-1 ; 1 ; q)\right]+\left[\operatorname{ELi}_{0 ; 0}\left(1 ; q^{-1} ; q\right)+\operatorname{ELi}_{0 ; 0}\left(-1 ; q^{-1} ; q\right)\right] \\
& -\left[\operatorname{ELi}_{-1 ; 0}\left(1 ; q^{-1} ; q\right)+\operatorname{ELi}_{-1 ; 0}\left(-1 ; q^{-1} ; q\right)\right] .
\end{aligned}
$$

Furthermore, $1 / \mathbf{K}(z(x))$ is given by

$$
\begin{aligned}
\frac{1}{\mathbf{K}\left(k^{2}\right)}= & \frac{2}{\pi \eta^{12}(\tau)}\left\{\frac{5}{48}\left\{1-24 \mathrm{ELi}_{-1 ; 0}(1 ; 1 ; q)-4\left[1-24 \mathrm{ELi}_{-1 ; 0}\left(1 ; 1 ; q^{4}\right)\right]\right\}\right. \\
& \times\left\{-1-4\left[\operatorname{ELi}_{0 ; 0}\left(-1 ; 1 / q ; q^{2}\right)-4 \mathrm{ELi}_{-1 ; 0}\left(-1 ; 1 / q ; q^{2}\right)+4 \mathrm{ELi}_{-2 ; 0}\left(-1 ; 1 / q ; q^{2}\right)\right]\right\} \\
& -\frac{1}{16}\left\{5-4\left[\operatorname{ELi}_{0 ; 0}\left(-1 ; 1 / q ; q^{2}\right)-8 \mathrm{ELi}_{-1 ; 0}\left(-1 ; 1 / q ; q^{2}\right)+24 \mathrm{ELi}_{-2 ; 0}\left(-1 ; 1 / q ; q^{2}\right)\right.\right. \\
& \left.\left.-32 \mathrm{ELi}_{-3 ; 0}\left(-1 ; 1 / q ; q^{2}\right)+16 \mathrm{ELi}_{-4 ; 0}\left(-1 ; 1 / q ; q^{2}\right)\right]\right\}
\end{aligned}
$$

which is a modular function. Terms of this kind do not allow to integrate the formal power series in $q$, since the coefficients of the power series in $1 / \eta(\tau)$ are not known in closed form. Therefore, the expressions obtained in the present case are of a more general nature than those appearing in $[34,39,41]$. Another generalization appears due to the emergence of elliptic polylogarithm representations in which the parameters $x$ and $y$ become $q$-dependent.

\section{Conclusions}

For single scale processes in the massless and massive cases to two-loop order one always has found iterative integral solutions over the alphabets given in [1,5] and the special numbers [50]. This applies also to the massless case at 3-loop order [58-60]. For the massive OMEs and Wilson coefficients in the asymptotic region $Q^{2} \gg m^{2}$, with $Q^{2}$ the virtuality of the deep-inelastic process and $m$ the heavy quark mass, in the case of the iterative integral solutions $[15,61-68]$ more general alphabets contribute as well for the nested sums, iterated integrals, and in some cases also for the special constants [2-4]. Beginning with 3-loop order one of the OMEs, $A_{Q g}^{(3)}$, $[69,70]$ contains also iterative non-iterative integrals caused by the emergence of the elliptic integrals of the first and second kind in some irreducible differential equations of second order. These are directly related to the differential equations studied in [24] for the 3-loop QCD corrections of the $\rho$-parameter.

We have shown that 3-loop anomalous dimensions can be computed in a massive environment in an automated way [49] from the $O(1 / \varepsilon)$ term of the unrenormalized OME $\hat{\hat{A}}_{Q g}$. Using 
the method of arbitrary large moments [51], despite the fact that the master integrals contain elliptic terms at $O\left(\varepsilon^{0}\right)$ and higher, an assembly of all terms for fixed moments is possible, such that the final difference equations factorize at first order. A solution using the packages Sigma, EvaluateMultiSums, SumProduction and HarmonicSums is then possible.

This is likewise the case for 18 out of 28 color and $\zeta$-terms for the $O\left(\varepsilon^{0}\right)$ term as well, cf. [70]. For the remaining color and $\zeta$-terms in part of the integrals the complete elliptic integrals of the first and second kind emerge, as well as integrals of other letters over them. The corresponding differential equations are related to those emerging in the case of the 3-loop QCD corrections to the $\rho$-parameter by a variable transformation. We have outlined their solution in Section 3 leading to iterated non-iterative integral solutions. They possess analytic, fast converging series solutions around $x=0,1$, [24]. We have also studied the associated $q$-series solutions. Here generalizations of previously studied elliptic solutions $[34,39,41]$ occur. Due to the emergence of Dedekind- $\eta$ factors $\propto 1 / \eta^{k}(\tau), k \in \mathbb{N}, k>0$, in front of modular forms expressed in (products of) LambertEisenstein series, it is in general not possible to find a diagonalization of the corresponding integrals, finally expressed in terms of elliptic polylogarithms. However, elliptic polylogarithms can be widely used as a framework for the representation of the different building blocks emerging in the solution, also allowing their parameters $x$ and $y,(1.10)$, to depend on $q$.

\section{References}

[1] E. Remiddi and J.A.M. Vermaseren, Int. J. Mod. Phys. A 15 (2000) 725 [hep-ph/9905237].

[2] J. Ablinger, J. Blümlein and C. Schneider, J. Math. Phys. 54 (2013) 082301 [arXiv:1302.0378 [math-ph]].

[3] J. Ablinger, J. Blümlein and C. Schneider, J. Math. Phys. 52 (2011) 102301 [arXiv:1105.6063 [math-ph]].

[4] J. Ablinger, J. Blümlein, C.G. Raab and C. Schneider, J. Math. Phys. 55 (2014) 112301 [arXiv:1407.1822 [hep-th]].

[5] J.A.M. Vermaseren, Int. J. Mod. Phys. A 14 (1999) 2037 [hep-ph/9806280]; J. Blümlein and S. Kurth, Phys. Rev. D 60 (1999) 014018 [arXiv:hep-ph/9810241].

[6] M. Karr, J. ACM 28 (1981) 305.

[7] C. Schneider, RISC, Johannes Kepler University, Linz technical report 01-17 (2001).

[8] C. Schneider, An. Univ. Timisoara Ser. Mat.-Inform. 42 (2004) 163; J. Differ. Equations Appl. 11 (2005) 799; Appl. Algebra Engrg. Comm. Comput. 16 (2005) 1.

[9] C. Schneider, J. Algebra Appl. 6 (2007) 415.

[10] C. Schneider, Motives, Quantum Field Theory, and Pseudodifferential Operators (Clay Mathematics Proceedings Vol. 12 ed. A. Carey, D. Ellwood, S. Paycha and S. Rosenberg,(Amer. Math. Soc) (2010), 285 [arXiv:0904.2323].

[11] C. Schneider, Ann. Comb. 14 (2010) 533 [arXiv:0808.2596].

[12] C. Schneider, in: Computer Algebra and Polynomials, Applications of Algebra and Number Theory, J. Gutierrez, J. Schicho, M. Weimann (ed.), Lecture Notes in Computer Science (LNCS) 8942 (2015), 157[arXiv:13077887 [cs.SC]]. 
[13] C. Schneider, J. Symbolic Comput. 43 (2008) 611 [arXiv:0808.2543v1]; J. Symb. Comput. 72 (2016) 82 [arXiv:1408.2776 [cs.SC]]; J. Symb. Comput. 80 (2017) 616 [arXiv:1603.04285 [cs.SC]].

[14] C. Schneider, Ann. Comb. 9(1) (2005) 75;

S.A. Abramov and M. Petkovšek, J. Symbolic Comput., 45(6) (2010) 684;

C. Schneider, Structural theorems for symbolic summation, Appl. Algebra Engrg. Comm. Comput., 21(1) (2010) 1;

C. Schneider, In: Symbolic and Numeric Algorithms for Scientific Computing (SYNASC), 2014, 15th International Symposium, F. Winkler, V. Negru, T. Ida, T. Jebelean, D. Petcu, S. Watt, D. Zaharie (ed.), (2015) pp. 26; IEEE Computer Society, arXiv:1412.2782v1 [cs.SC].

[15] J. Ablinger, A. Behring, J. Blümlein, A. De Freitas, A. von Manteuffel and C. Schneider, Comput. Phys. Commun. 202 (2016) 33 [arXiv:1509.08324 [hep-ph]].

[16] C. Schneider, Sém. Lothar. Combin. 56 (2007) 1, article B56b.

[17] C. Schneider, Computer Algebra in Quantum Field Theory: Integration, Summation and Special Functions Texts and Monographs in Symbolic Computation eds. C. Schneider and J. Blümlein (Springer, Wien, 2013) 325, arXiv:1304.4134 [cs.SC].

[18] J. Ablinger, J. Blümlein, S. Klein and C. Schneider, Nucl. Phys. Proc. Suppl. 205-206 (2010) 110 [arXiv:1006.4797 [math-ph]];

J. Blümlein, A. Hasselhuhn and C. Schneider, PoS (RADCOR 2011) 032 [arXiv:1202.4303 [math-ph]];

C. Schneider, J. Phys. Conf. Ser. 523 (2014) 012037 [arXiv:1310.0160 [cs.SC]].

[19] J. Ablinger, Computer Algebra Algorithms for Special Functions in Particle Physics, Ph.D. Thesis, J. Kepler University Linz, 2012, arXiv:1305.0687 [math-ph].

[20] J. Ablinger, PoS (LL2014) 019; Computer Algebra Algorithms for Special Functions in Particle Physics, Ph.D. Thesis, J. Kepler University Linz, 2012, arXiv:1305.0687 [math-ph].

[21] K. Heun, Math. Ann. 33 (1889) 161;

A. Ronveaux, ed., Heun's differential equations, (The Clarendon Press Oxford, Oxford, 1995); J. Ablinger et al., in preparation.

[22] J. Blümlein, S. Klein, C. Schneider and F. Stan, J. Symbolic Comput. 47 (2012) 1267 [arXiv:1011.2656 [cs.SC]].

[23] E.W. Barnes, Proc. Lond. Math. Soc. (2) 6 (1908) 141; Quart. Journ. Math. 41 (1910) 136; H. Mellin, Math. Ann. 68 (1910) 305.

[24] J. Ablinger, J. Blümlein, A. De Freitas, M. van Hoeij, E. Imamoglu, C. G. Raab, C.-S. Radu and C. Schneider, arXiv:1706.01299 [hep-th].

[25] A. Sabry, Nucl. Phys. 33 (1962) 401.

[26] D.J. Broadhurst, J. Fleischer and O.V. Tarasov, Z. Phys. C 60 (1993) 287 [hep-ph/9304303];

F.A. Berends, M. Buza, M. Böhm and R. Scharf, Z. Phys. C 63 (1994) 227;

S. Bauberger, M. Böhm, G. Weiglein, F.A. Berends and M. Buza, Nucl. Phys. Proc. Suppl. 37B (1994) no.2, 95 [hep-ph/9406404];

S. Bauberger and M. Böhm, Nucl. Phys. B 445 (1995) 25 [hep-ph/9501201].

[27] M. Caffo, H. Czyz and E. Remiddi, Nucl. Phys. B 634 (2002) 309 [hep-ph/0203256].

[28] S. Laporta and E. Remiddi, Nucl. Phys. B 704 (2005) 349 [hep-ph/0406160]. 
[29] S. Pozzorini and E. Remiddi, Comput. Phys. Commun. 175 (2006) 381 [hep-ph/0505041]; S. Groote, J.G. Körner and A.A. Pivovarov, Annals Phys. 322 (2007) 2374 [hep-ph/0506286]; B.A. Kniehl, A.V. Kotikov, A. Onishchenko and O. Veretin, Nucl. Phys. B 738 (2006) 306 [hep-ph/0510235]; M. Caffo, H. Czyz, M. Gunia and E. Remiddi, Comput. Phys. Commun. 180 (2009) 427 [arXiv:0807.1959 [hep-ph]].

[30] U. Aglietti, R. Bonciani, L. Grassi and E. Remiddi, Nucl. Phys. B 789 (2008) 45 [arXiv:0705.2616 [hep-ph]].

[31] D.H. Bailey, J.M. Borwein, D. Broadhurst and M.L. Glasser, J. Phys. A 41 (2008) 205203 [arXiv:0801.0891 [hep-th]].

[32] D. Broadhurst, Elliptic integral evaluation of a Bessel moment by contour integration of a lattice Green function, arXiv:0801.4813 [hep-th].

[33] S. Müller-Stach, S. Weinzierl and R. Zayadeh, Commun. Num. Theor. Phys. 6 (2012) 203 [arXiv:1112.4360 [hep-ph]];

S. Groote, J.G. Körner and A.A. Pivovarov, Eur. Phys. J. C 72 (2012) 2085 [arXiv:1204.0694 [hep-ph]];

S. Müller-Stach, S. Weinzierl and R. Zayadeh, Commun. Math. Phys. 326 (2014) 237 [arXiv:1212.4389 [hep-ph]].

[34] S. Bloch and P. Vanhove, J. Number Theor. 148 (2015) 328, [arXiv:1309.5865 [hep-th]].

[35] L. Adams, C. Bogner and S. Weinzierl, J. Math. Phys. 54 (2013) 052303 [arXiv:1302.7004 [hep-ph]]; E. Remiddi and L. Tancredi, Nucl. Phys. B 880 (2014) 343 [arXiv:1311.3342 [hep-ph]].

[36] L. Adams, C. Bogner and S. Weinzierl, J. Math. Phys. 56 (2015) no.7, 072303 [arXiv:1504.03255 [hep-ph]].

[37] L. Adams, C. Bogner and S. Weinzierl, J. Math. Phys. 57 (2016) no.3, 032304 [arXiv:1512.05630 [hep-ph]].

[38] L. Adams, C. Bogner and S. Weinzierl, PoS (LL2016) 033 [arXiv:1606.09457 [hep-ph]]; S. Bloch, M. Kerr, P. Vanhove, Compos. Math. 151 (2015) 2329. [arXiv:1406.2664[hep-th]]; Local mirror symmetry and the sunset Feynman integral, arXiv:1601.08181 [hep-th].

[39] L. Adams, C. Bogner and S. Weinzierl, J. Math. Phys. 55 (2014) no.10, 102301 [arXiv:1405.5640 [hep-ph]].

[40] E. Remiddi and L. Tancredi, Nucl. Phys. B 907 (2016) 400 [arXiv:1602.01481 [hep-ph]].

[41] L. Adams, C. Bogner, A. Schweitzer and S. Weinzierl, J. Math. Phys. 57 (2016) 122302 [arXiv:1607.01571 [hep-ph]].

[42] J. Brödel, C.R. Mafra, N. Matthes and O. Schlotterer, JHEP 1507 (2015) 112 [arXiv:1412.5535 [hep-th]];

R. Bonciani, V. Del Duca, H. Frellesvig, J. M. Henn, F. Moriello and V. A. Smirnov, JHEP 1612 (2016) 096 [arXiv:1609.06685 [hep-ph]].

[43] J. Serre, A Course in Arithmetic, (Springer, Berlin, 1973).

[44] J.M. Borwein and P.B. Borwein, $\pi$ and the AGM, (Wiley-Interscience, New York, 1987). 
[45] R. Dedekind, Journal für die reine und angewandte Mathematik (Crelle) 83 (1877) 265; in: Gesammelte mathematische Werke, ed. R. Fricke, E. Noether, Ø. Ore, (Viehweg und Sohn, Braunschweig, 1930) XIV. Schreiben an Herrn Borchhardt über die Theorie der elliptischen Modulfunktionen, pp. 174; Erläuterungen zu den Fragmenten XXVIII, pp. 454 (pp. 486), in: B. Riemann, Gesammelte Mathematische Werke, Wissenschaftlicher Nachlaß und Nachträge, nach der Ausgabe von H. Weber und R. Dedekind neu herausgegeben von R. Narasimhan, (Springer, Berlin, 1990) und (Teubner, Leipzig, 1990).

[46] J.H. Lambert, Anlage zur Architectonic oder Theorie des Ersten und des Einfachen in der philosophischen und der mathematischen Erkenntniß, (Johann Friedrich Hartknoch, Riga, 1771), 2, $\S 875$, pp. 506.

[47] B. Eisenstein, Mathematische Abhandlungen, (Berlin, G. Reimer, 1847), pp. 213-334; Journal für die reine und angewandte Mathematik (Crelle) 35 (1847) 153;

[48] H. Cohen and F. Strömberg, Modular Forms, A Classical Approach, Graduate Studies in Mathematics 179 (AMS, Providence,RI, 2017).

[49] J. Ablinger, A. Behring, J. Blümlein, A. De Freitas, A. von Manteuffel and C. Schneider, Nucl. Phys. B 922 (2017) 1 [arXiv:1705.01508 [hep-ph]].

[50] J. Blümlein, D.J. Broadhurst and J.A.M. Vermaseren, Comput. Phys. Commun. 181 (2010) 582 [arXiv:0907.2557 [math-ph]].

[51] J. Blümlein and C. Schneider, Phys. Lett. B 771 (2017) 31 [arXiv:1701.04614 [hep-ph]].

[52] M. Kauers, Guessing Handbook, Technical Report RISC 09-07, JKU Linz.

[53] J. Blümlein, M. Kauers, S. Klein and C. Schneider, Comput. Phys. Commun. 180 (2009) 2143 [arXiv:0902.4091 [hep-ph]].

[54] J. Grigo, J. Hoff, P. Marquard and M. Steinhauser, Nucl. Phys. B 864 (2012) 580 [arXiv:1206.3418 [hep-ph]].

[55] E. Imamoglu and M. van Hoeij, J. Symbolic Comput. 83 (2017) 245 [arXiv:1606.01576 [cs.SC]].

[56] K. Takeuchi, J. Fac. Sci, Univ. Tokyo, Sect 1A 24 (1977) 201.

[57] F.G. Tricomi, Elliptische Funktionen, (Geest \& Portig, Leipzig, 1948); übersetzt und bearbeitet von M. Krafft;

E.T. Whittaker and G.N. Watson, A Course of Modern Analysis, (Cambridge University Press, Cambridge, 1996); reprint of the 4th edition (1927).

[58] A. Vogt, S. Moch and J.A.M. Vermaseren, Nucl. Phys. B 691 (2004) 129 [hep-ph/0404111].

[59] S. Moch, J.A.M. Vermaseren and A. Vogt, Nucl. Phys. B 688 (2004) 101 [hep-ph/0403192].

[60] J.A.M. Vermaseren, A. Vogt and S. Moch, Nucl. Phys. B 724 (2005) 3 [hep-ph/0504242].

[61] J. Ablinger, A. Behring, J. Blümlein, A. De Freitas, A. Hasselhuhn, A. von Manteuffel, M. Round, C. Schneider, and F. Wißbrock, Nucl. Phys. B 886 (2014) 733 [arXiv:1406.4654 [hep-ph]].

[62] J. Ablinger, A. Behring, J. Blümlein, A. De Freitas, A. von Manteuffel and C. Schneider, Nucl. Phys. B 890 (2014) 48 [arXiv:1409.1135 [hep-ph]].

[63] J. Ablinger, J. Blümlein, A. De Freitas, A. Hasselhuhn, A. von Manteuffel, M. Round and C. Schneider, Nucl. Phys. B 885 (2014) 280 [arXiv:1405.4259 [hep-ph]]. 
[64] J. Ablinger, J. Blümlein, A. De Freitas, A. Hasselhuhn, A. von Manteuffel, M. Round, C. Schneider and F. Wißbrock, Nucl. Phys. B 882 (2014) 263 [arXiv:1402.0359 [hep-ph]].

[65] J. Ablinger, J. Blümlein, S. Klein, C. Schneider and F. Wißbrock, Nucl. Phys. B 844 (2011) 26 [arXiv:1008.3347 [hep-ph]].

[66] J. Blümlein, A. Hasselhuhn, S. Klein and C. Schneider, Nucl. Phys. B 866 (2013) 196 [arXiv:1205.4184 [hep-ph]].

[67] A. Behring, I. Bierenbaum, J. Blümlein, A. De Freitas, S. Klein and F. Wißbrock, Eur. Phys. J. C 74 (2014) no.9, 3033 [arXiv:1403.6356 [hep-ph]].

[68] J. Ablinger et al., DESY 15-112.

[69] I. Bierenbaum, J. Blümlein and S. Klein, Nucl. Phys. B 820 (2009) 417 [arXiv:0904.3563 [hep-ph]]; J. Blümlein, S. Klein and B. Tödtli, Phys. Rev. D 80 (2009) 094010 [arXiv:0909.1547 [hep-ph]].

[70] J. Ablinger, A. Behring, J. Blümlein, A. De Freitas, A. von Manteuffel and C. Schneider, Heavy Flavor Wilson Coefficients in Deep-Inelastic Scattering: Recent Results, arXiv:1711.07957 [hep-ph]. 